\title{
The effects of perceptual condition on proofreading for misspellings
}

\author{
ALICE F. HEALY, VICKI J. VOLBRECHT, and TERRY R. NYE \\ University of Colorado, Boulder, Colorado
}

\begin{abstract}
In two experiments, subjects proofread text in which misspelled words were created by replacing a single letter with another one. The following perceptual conditions were included: clear-cut photocopies of typewritten text, clear-cut text presented on a CRT screen, ditto copies that varied in legibility, and text that included extraneous noise characters superimposed on some letters. In all conditions, subjects adopted a hierarchical feature test that gave first priority to resolving letter envelope and second priority to discriminating other letter features. When clear-cut text with no extraneous noise was proofread, subjects used a sophisticated-guessing decision rule that tolerated misspellings involving missing letter features (as when $\mathrm{c}$ replaced $\mathrm{e}$ ) but not added ones (as when e replaced c). This asymmetrical rule was modified, however, when subjects were exposed to text that included extraneous noise that was confusable with the letter features. In those circumstances, subjects adopted a decision rule that tolerated misspellings in volving missing features or any added features that resembled the noise.
\end{abstract}

In previous experiments, Healy (1981) provided evidence that proofreaders searching for misspellings employ a particular pair of information processing rules: A hierarchical feature test (see, e.g., Massaro \& Schmuller, 1975) coupled with a sophisticated-guessing decision rule (see, e.g., Broadbent, 1967; Lindsay \& Norman, 1977; and Rumelhart \& Siple, 1974). By the hierarchical feature test, subjects give greatest weight to resolving the envelope of a letter and only secondarily discriminate additional visual features. Letter envelope is defined as "the smallest enclosing polygon without indentations" (Bouma, 1971, p. 463). Healy (1981, p. 459) noted that her results provide strong evidence for a hierarchy of levels of discrimination but do not provide any information concerning the temporal sequence in which those levels are processed. In accord with the work of Lupker (1979), it seems likely that subjects first resolve letter envelope and then process additional features, but it is also possible, given Healy's (1981) findings, that the two levels of discrimination are processed in parallel, with

This research was supported by NSF Grant BNS80-25020 to the Institute of Cognitive Science at the University of Colorado and by BRSG Grant RR07013-16 awarded to the University of Colorado by the Biomedical Research Support Grant Program, Division of Research Resources, National Institutes of Health. The authors are indebted to Adam Drewnowski for his helpful suggestions that led to the hyphen and slash conditions of Experiment 2, to Timothy McNamara and William Oliver for help with the computer programming involved in the CRT condition of Experiment 1, to Michael Wertheimer for helpful discussions about this research, to Mary Ann Gundel for help in using the ditto equipment for Experiment 2, and to Steven Keele and four anonymous reviewers for their helpful comments on earlier versions of this article. The authors' mailing address is: Department of Psychology, University of Colorado, Campus Box 345, Boulder, Colorado 80309. more weight given to those features defining envelope than to other features. If the envelope of the letter conforms to their expectations, the proofreaders adopt a sophisticated-guessing decision rule in which they tolerate the absence of letter features but are intolerant of additional features. According to the sophisticatedguessing decision rule, subjects tolerate missing features because they base their recognition decisions on partial information that they have extracted from the stimulus. The letter features that have been identified serve as constraints on the letter-recognition responses, so that only letters that contain the identified features are candidates for recognition. A related alternative formulation, which cannot readily be distinguished from sophisticated guessing, is that subjects tolerate missing features because these features are filled in by the perceptual processes, in accord with the completion phenomenon noted by Gestalt psychologists (see, e.g., Koffka, 1935).

These information processing rules were supported by two related findings in the study by Healy (1981): (1) Subjects searching for misspelled words made many more proofreading errors when a correct letter was replaced by another letter that had the same visual features except for one that was missing (as when e was replaced by c) than when the letter substitution was made in the reverse order (as when $\mathrm{c}$ was replaced by e). (2) This large asymmetry was found only when the correct letter and the one replacing it had the same envelope (as with $\mathrm{c}$ and $\mathrm{e}, \mathrm{C}$ and $\mathrm{G}, \mathrm{c}$ and $\mathrm{o}$, and $\mathrm{C}$ and 0 ); it was not found when the two letters had different envelopes, even when they also differed by only a single visual feature (as with $\mathrm{n}$ and $\mathrm{h}$ ). Letter envelope was implicated rather than letter shape, which simply differentiates letters with ascending strokes (e.g., d), those 
with descending strokes (e.g., q), and those with neither (e.g., o), because of the observation that c/e substitutions yielded more errors in lowercase than in uppercase. In both typecases, those two letters have the same shape, but they have the same envelope only in lowercase $(\mathrm{c} / \mathrm{e})$, not in uppercase $(\mathrm{C} / \mathrm{E})$. Furthermore, the asymmetrical decision rule was not found for $E / F$ or $P / R$ substitutions, and the letters in these pairs have the same shape in terms of ascending and descending strokes but have different envelopes. The simple three-category shape classification overlooks the configural properties of words printed in uppercase characters (see Garner, 1981), whereas the notion of envelope seems to capture the relevant variables.

A critical question left unanswered by previous work is the extent to which the information processing rules adopted by proofreaders are determined by the format and quality of the printed text that they are given to read. In the earlier experiments, the proofreaders read photocopies of typewritten text. Although the copies were clear and did not include any missing line segments, subjects may have interpreted misspellings involv. ing feature deletions as flawed print rather than as letter substitutions, because of their vast experience outside the laboratory with poor-quality photocopies that contained many missing line segments but rarely any extra segments. Perhaps under different perceptual conditions, in which subjects do not expect to find missing line segments or do expect additional irrelevant line segments instead of missing ones, the subjects' information processing strategies would be different.

The hypothesis that different proofreading error patterns would be found under different perceptual conditions is supported for tachistoscopic letter identification by the findings of Garner and Haun (1978). The pattern of identification errors in their task depended on the type of perceptual limitation they imposed: With a state limitation, in which stimulus duration was reduced, letters with more features were more often mistaken for those with fewer features than vice versa. The reverse asymmetric pattern of confusions occurred, however, with a process limitation, in which the letter patterns were distorted by both adding and deleting line segments.

One reason why it is important to test for the influence of perceptual condition on processing strategy is that a number of previous investigations have yielded evidence inconsistent with the sophisticated-guessing decision rule, and those investigations used perceptual conditions that differed from the ones used in the proofreading study by Healy (1981). For example, in a tachistoscopic letter-recognition experiment, Lindsay and Norman (1977, p. 267) found more occasions when $\mathrm{C}$ was reported as $\mathrm{G}$ than when $\mathrm{G}$ was reported as $C$, in accord with sophisticated guessing, but they did not find, for example, that $O$ was reported as $Q$ more than Q was reported as O. Massaro and Schmuller (1975) interpreted the asymmetries that were discovered in previous studies as artifacts of the subjects' response biases (e.g., subjects may have had a bias to report $G$ ). In fact, in an analysis of a tachistoscopic letter-recognition experiment, Massaro and Schmuller corrected for possible response bias by computing a $\mathrm{d}^{\prime}$ index and found that the asymmetries previously evident in the confusion matrix were thereby eliminated. Healy (1981) controlled for response bias in her study by comparing passages typed in lowercase and uppercase characters. Since the asymmetries she observed were typically much larger in one typecase than in the other (i.e., the asym. metry for $c$ and e was greater in lowercase, whereas that for $\mathrm{c}$ and $\mathrm{g}$ was greater in uppercase), they cannot be attributed solely to the subjects' biases to report specific letters, and therefore they can be taken as support for sophisticated guessing. Nevertheless, it is reasonable to hypothesize that the sophisticated-guessing rule found by Healy would be evident under some perceptual conditions but not others, since the perceptual conditions of the Healy study were quite different from those in the studies of tachistoscopic letter report.

The aim of the present study was to determine whether the pattern of proofreading errors made in searching for misspelled words would be affected by perceptual condition. In Experiment 1, the text to be proofread was presented either on paper, as in the earlier investigation by Healy (1981), or on a CRT display screen attached to a computer terminal, which is a condition more similar to those in the earlier studies of tachistoscopic letter recognition. If proofreaders tolerate misspellings involving missing features only when they can interpret those misspellings as flawed printing due to a poor photocopying process, then such a tolerance should be found in the paper condition but not in the CRT condition. Although subjects may expect to see noisy CRT displays because of their previous experience outside the laboratory with poor television reception, they. should be no more likely on the basis of such experience to expect missing features than added features with the CRT display. On the other hand, if the sophisticated-guessing rule is a general proofreading strategy, then similar patterns of proofreading errors should be obtained in the paper and CRT conditions. In Experiment 2, the text to be proofread was always presented on paper, but irrelevant line segments were superimposed on the text in some conditions. Subjects might be led under those circumstances to tolerate misspellings involving additional visual features as well as those involving missing features.

The information processing strategies adopted by proofreaders in these conditions were examined by using the method and text material previously employed by Healy (1981, Experiment 3). The misspellings included in the text were created in all cases by replacing a single letter in a word with another one (see Healy, 1980, and Holbrook, 1978, for other uses of this substitution-proofreading procedure). In order to control for response bias and for linguistic factors such as word function and letter and word frequency, the text to be proofread was typed in two different formats, 
one with all uppercase letters and one with lowercase letters. It was reasoned that only visual factors, not linguistic ones, would be affected by changes in typecase. The letter substitutions that were used involved three different letter pairs, each of which included letters that had the same envelope and differed by a single letter feature ${ }^{1}$ when printed in one typecase. The letters in one pair $(\mathrm{c} / \mathrm{e})$ had the same envelope in lowercase but not in uppercase; those in the second pair $(\mathrm{c} / \mathrm{g})$ had the same envelope in uppercase but not in lowercase; and those in the third pair $(\mathrm{c} / \mathrm{o})$ had the same envelope in both typecases. Evidence for the salience of letter envelope was gathered by examining the effect of typecase: A hierarchical feature test giving greatest weight to the resolution of envelope was supported by the finding of more proofreading errors for the c/e substitutions in lowercase than in uppercase, more proofreading errors for $\mathrm{c} / \mathrm{g}$ substitutions in uppercase than in lowercase, and comparable percentages of proofreading errors for $\mathrm{c} / \mathrm{o}$ substitutions in the two typecases. Evidence for the sophisticated-guessing decision rule was then gathered by examining the effect of the direction of letter substitution: An asymmetrical decision rule in which subjects tolerate missing visual features but are intolerant of additional features was supported by the finding of more proofreading errors for substitutions involving the deletion of a single short line segment (c for $\mathrm{e}, \mathrm{C}$ for $\mathrm{G}$, c for $\mathrm{O}$, or $\mathrm{C}$ for $\mathrm{O}$ ) than for substitutions involving the addition of a line segment ( $e$ for $c$, $\mathrm{G}$ for $\mathrm{C}$, o for $\mathrm{c}$, or $\mathrm{O}$ for $\mathrm{C}$ ).

\section{EXPERIMENT 1}

Two perceptual conditions were compared in Experiment 1: The text was displayed on sheets of paper, having been reproduced for each subject by photocopying from typewritten originals, or on a CRT screen. In order to monitor the subjects' responses in the CRT condition, the text was displayed five words at a time for a fixed duration, and the subjects indicated each misspelling by depressing a response button. The text was also presented in five-word segments in the paper condition, one segment per line, but the subjects responded in that condition by highlighting the misspelled words with a yellow marker.

\section{Method}

Subjects. Twenty-four undergraduate men and women from the University of Colorado participated for introductory psychology course credit.

Materials and apparatus. The same 1,225-word prose passage employed by Healy (1981) was used here. This passage was an excerpt from an elementary textbook on social psychology by Aronson (1972). As in Experiment 3 of the earlier study by Healy (1981), two versions of the passage were used, with 90 misspellings in each version. The two passage versions were identical except that one was typed with standard capitalization and all substituted letters in lowercase ("lowercase"), whereas the other was typed with all capital letters ("uppercase"). The misspellings were inserted with the following four constraints:
(1) No misspellings were placed in the first 10 or final 15 words of the passage. The remaining 1,200 words were divided into 12010 -word blocks. One word was misspelled in 6 of every 8 successive blocks.

(2) All misspellings were formed by replacing one letter in the word with a different letter. None of these substitutions yielded another correctly spelled English word.

(3) There were six types of substitutions: e for $c, c$ for $e$ $\mathrm{g}$ for $\mathrm{c}, \mathrm{c}$ for $\mathrm{g}$, o for $\mathrm{c}$, and $\mathrm{c}$ for $\mathrm{o}$. Each of the six types occurred 15 times in the passage and once in every eight blocks. See Table 1 for examples of the six types of misspellings, printed in all lowercase letters and printed in all uppercase letters.

(4) The two types of substitutions involving the same letter pair (e.g., e for $c$ and $c$ for e) were equated as closely as possible with respect to three variables: the frequency in English of the correctly spelled word, as determined by Kưera and Francis (1967), the length of the word, and the location of the substituted letter in the word. Word function was not specifically controlled, but most of the substitutions occurred within content words rather than within function words. See Healy (1981) for further details concerning these constraints.

For the paper condition, both typecase versions of the passage were typed with single spacing in a single five-word column on five different sheets of paper. The typing was accomplished by means of a Diablo 1620 typewriter terminal with an Elite 12 print wheel and a black multistrike (film) ribbon. The passage was photocopied for distribution to the subjects.

The passages were displayed for subjects in the CRT condition on a Visual 200 terminal display screen linked to a PDP-11/ 03 computer system. The first letter in a display was positioned 12 lines down and 20 spaces over from the upper left-hand corner of the screen, which included 24 lines and 80 spaces across. Only a single five-word line was displayed at any given instant, and each line was shown for a fixed amount of time. The display duration was $2,500 \mathrm{msec}$ for the first 2 subjects and $3,000 \mathrm{msec}$ for the remaining 10 subjects. The duration was increased because of the relatively large number of false-alarm errors for the first 2 subjects (see the discussion below of false alarms).

Subjects made their responses in the paper condition by writing with a yellow highlighter marker and in the CRT condition by depressing a response button connected to the computer. A button press by the subject stopped a timer in the computer that was initiated at the onset of each 5-word display, so that the subjects' response latencies were recorded. Since the passage was constructed so that there was never more than one substitution in every set of 10 successive words, there was never more than one misspelling in each 5 -word display. Therefore, a response made during a display containing a misspelling was scored as correct, whereas one made during a display with no misspelling was scored as a false alarm.

Design. A four-way mixed design was employed with one between-subjects factor (perceptual condition) and three withinsubjects factors (passage typecase, letter pair, and direction of substitution). Twelve subjects were tested in each of the two

Table 1

Examples of Misspellings Used in Experiments

\begin{tabular}{cll}
$\begin{array}{c}\text { Substitution } \\
\text { Type }\end{array}$ & Lowercase & \multicolumn{1}{c}{ Uppercase } \\
\hline c to e & aecordingly & AECORDINGLY \\
$\mathrm{e}$ to $\mathrm{c}$ & potcnt & POTCNT \\
$\mathrm{c}$ to $o$ & beoome & BEOOME \\
o to $\mathrm{c}$ & perscn & PERSCN \\
$\mathrm{c}$ to $\mathrm{g}$ & onge & ONGE \\
$\mathrm{g}$ to $\mathrm{c}$ & strancer & STRANCER \\
\hline
\end{tabular}


perceptual conditions-CRT and paper. Six subjects in each condition received the lowercase passage version first and the uppercase version second. The other six subjects received the typecase versions in the opposite order. The assignment of subjects to conditions and presentation orders was determined on the basis of time of arrival for testing according to a fixed rotation.

Procedure. Subjects were tested individually in a private, quiet room. Instructions were typewritten and photocopied for all subjects, but the instructions differed somewhat for the two experimental conditions.

In the paper condition, subjects were told that they were to read two prose passages at their "normal reading speed," but whenever they came to a spelling error they were to highlight it with the yellow marker provided to them. The subjects were told that if at any time they realized that they had missed a spelling error in a previous word, they should not retrace their steps to highlight it and that they should not slow down their reading speed or reread the text in order to be overcautious about catching the spelling errors. The subjects were further instructed to read the two passages in the order in which the passages were stapled together and not to refer to the first passage when reading the second.

In the CRT condition, subjects were told that they would see two prose passages shown five words per display on the computer terminal screen for a fixed amount of time. They were instructed to press the response button as quickly as possible whenever they noticed a spelling error. The subjects were told that if at any time they realized that they had missed a spelling error in a previous word no longer on the screen, they should not press the button or it would be scored as a mistake by the computer. The subjects were not warned, however, that there would never be more than one misspelling in every five-word display. The subjects were told to respond quickly and carefully, since both their speed and accuracy were scored by the computer. When subjects finished reading the first passage, the computer program was restarted by the experimenter to present the second passage.

\section{Results and Discussion}

Misses. The number of misspellings not detected for each type of misspelling in each passage typecase was computed for each subject. The data from both presentation orders were combined, and the mean percentages of proofreading errors made by subjects were computed as a function of condition, passage typecase, and substitution type and are displayed in Table 2 . The standard errors of the values in the table, as determined by analyses of variance, are $2.5 \%$ for the paper condition and $1.7 \%$ for the CRT condition.

A multifactorial repeated-measures analysis of variance was conducted on these data. The only betweensubjects factor included was perceptual condition (paper, CRT). The three within-subjects factors were passage typecase (lowercase, uppercase), letter pair $(\mathrm{c} / \mathrm{e}, \mathrm{c} / \mathrm{g}, \mathrm{c} / \mathrm{o})$, and direction of substitution (additional feature, deleted feature). The significant results of the analysis are summarized in Table 3.

Both perceptual conditions showed evidence for the hierarchical feature test and the asymmetrical decision rule. There were also some interesting differences between the two conditions that may be explained in terms of differences in the type font in which the passages were printed in the two conditions.

Evidence for the hierarchical feature test that gives first priority to resolving letter envelope was provided by the significant interaction between passage typecase and letter pair. As expected and as found in the earlier study by Healy (1981), more proofreading errors were made on the lowercase version (13.9\%) than on the uppercase one $(6.0 \%)$ for $\mathrm{c} / \mathrm{e}$, more proofreading errors were made on uppercase $(18.9 \%)$ than on lowercase $(10.4 \%)$ for $\mathrm{c} / \mathrm{g}$, and the percentages of proofreading errors in lowercase $(8.6 \%)$ and uppercase $(6.4 \%)$ were much closer for $\mathrm{c} / \mathrm{o}$.

The asymmetrical decision rule was supported by the finding of significantly more proofreading errors when

Table 2

Percentages of Proof reading Errors in Experiment 1 as a Function of Perceptual Condition, Passage, Typecase, and Substitution Type

\begin{tabular}{|c|c|c|c|c|c|c|c|}
\hline \multirow{2}{*}{\multicolumn{2}{|c|}{ Perceptual Condition }} & \multicolumn{6}{|c|}{ Substitution Type } \\
\hline & & \multirow{2}{*}{$\begin{array}{c}\mathrm{c} \text { to } \mathrm{e} \\
4.4 \\
3.9\end{array}$} & \multirow{2}{*}{$\begin{array}{r}\text { e to } \mathrm{c} \\
\begin{array}{r}8.9 \\
3.9\end{array}\end{array}$} & \multirow{2}{*}{$\begin{array}{r}\text { c to } \mathrm{g} \\
6.7 \\
10.0\end{array}$} & \multirow{2}{*}{$\frac{\mathrm{g} \text { to } \mathrm{c}}{12.2}$} & \multirow{2}{*}{ 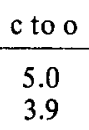 } & \multirow{2}{*}{$\begin{array}{r}0 \text { to } \mathrm{c} \\
8.9 \\
7.8\end{array}$} \\
\hline CRT & $\begin{array}{l}\text { Lowercase } \\
\text { Uppercase }\end{array}$ & & & & & & \\
\hline Paper & $\begin{array}{l}\text { Lowercase } \\
\text { Uppercase }\end{array}$ & $\begin{array}{r}10.0 \\
8.9\end{array}$ & $\begin{array}{r}32.2 \\
7.2\end{array}$ & $\begin{array}{r}7.2 \\
13.9\end{array}$ & $\begin{array}{l}15.6 \\
28.9\end{array}$ & $\begin{array}{l}6.7 \\
6.7\end{array}$ & $\begin{array}{r}13.9 \\
7.2\end{array}$ \\
\hline
\end{tabular}

Table 3

Significant Results of Four-Way Analysis of Variance on Error Data in Experiment 1

\begin{tabular}{lccc}
\multicolumn{1}{c}{ Effect } & Degrees of Freedom & F & p \\
\hline Letter Pair & 2,44 & 15.32 & .001 \\
Direction of Substitution & 1,22 & 61.00 & .001 \\
Condition x Letter Pair & 2,44 & 4.14 & .05 \\
Passage Typecase x Letter Pair & 2,44 & 35.21 & .001 \\
Passage Typecase x Direction of Substitution & 1,22 & 5.38 & .05 \\
Letter Pair x Direction of Substitution & 2,44 & 5.34 & .01 \\
Condition x Passage Typecase x Letter Pair & 2,44 & 5.73 & .01 \\
Condition x Passage Typecase x Direction of Substitution & 1,22 & 8.59 & .01 \\
Passage Typecase x Letter Pair x Direction of Substitution & 2,44 & 11.90 & .001 \\
\hline
\end{tabular}


features were deleted $(14.1 \%)$ than when they were added $(7.3 \%)$. Furthermore, the difference between the proofreading error rates for missing and added features was much larger when the correct letter and the one replacing it had the same envelope (ce, CG, co, $\mathrm{CO}$ ), as attested by the significant three-way interaction of passage typecase, letter pair, and direction of substitution.

One could argue that the pattern of proofreading errors noted here is not due to a general strategy but rather is an artifact of the particular misspellings included in the present passage. Only six different substitution types were used in this experiment, and these substitutions involved only four different letters (c, e, $\mathrm{g}, \mathrm{o}$ ), one of which (c) was used in each type. Perhaps subjects became especially attentive to these letters. However, this explanation cannot account for the fact that the asymmetry we found for $c$ and e was also obtained in an earlier study by Healy (1981, Experiment 2), in which $c$ was used less often than e, and the same asymmetry was not found in that study for substitution pairs that included letters with different envelopes (e.g., $n$ and $h$ ). Furthermore, this explanation, or any other explanation that makes reference to subject biases or to the linguistic properties of the letters rather than to their visual properties, cannot account for the large effects of typecase in this study: The fact that subjects made more proofreading errors on $c$ and e substitutions in lowercase and on $\mathrm{c}$ and $\mathrm{g}$ substitutions in uppercase cannot be explained in terms of the subjects' attention to certain letters but can be easily explained by the visual relationships among the letters.

Both conditions yielded similar patterns of proofreading errors; the four-way interaction of condition, passage typecase, letter pair, and direction of substitution was not significant $[F(2,44)=2.48, p>.05]$. Nevertheless, separate analyses of variance were conducted on each condition to confirm the similar patterns. For the paper condition, the hierarchical feature test was supported by a significant interaction of passage typecase and letter pair $[F(2,22)=24.13, p<.001]$. The asymmetrical decision rule was supported in the paper condition by the significant increase in proofreading errors for feature deletions compared with additions $[F(1,11)=48.46, p<.001]$, and the significant three-way interaction of direction of substitution, passage typecase, and letter pair $[F(2,22)=9.09, p<.01]$. Likewise, for the CRT condition, the hierarchical feature test was supported by a significant interaction of passage typecase and letter pair $[\mathrm{F}(2,22)=11.69$, $\mathrm{p}<.001]$. Support for the asymmetrical decision rule in the CRT condition came from finding significantly more proofreading errors on feature deletions than on feature additions $[\mathrm{F}(1,11)=16.61, \mathrm{p}<.01]$; however, the three-way interaction of direction of substitution, passage typecase, and letter pair did not reach standard levels of statistical significance $[F(2,22)=2.97, p<.10]{ }^{2}$

Although use of the same information processing rules was supported in the two perceptual conditions, there were some minor differences between them, as reflected in several significant interactions involving condition, including the interaction of condition and letter pair, the three-way interaction of condition, passage typecase, and letter pair, and the three-way interaction of condition, passage typecase, and direction of substitution. The greatest difference between the two conditions seems to involve the relative percentages of proofreading errors made on the different letter pairs, especially in the lowercase version. For lowercase, the ordering of letter pairs in terms of decreasing proofreading error percentages was $\mathrm{c} / \mathrm{g}, \mathrm{c} / \mathrm{o}$, and $\mathrm{c} / \mathrm{e}$ in the CRT condition, whereas in the paper condition it was $\mathrm{c} / \mathrm{e}, \mathrm{c} / \mathrm{g}$, and $\mathrm{c} / \mathrm{o}$. Examination of the type fonts used in the two conditions provides a possible explanation for these differences: Lowercase $c$ and $g$ are visually very similar in the type font used for the CRT condition, in which the tail on the $g$ is a short curved line, but not in that used for the paper condition, in which the tail on the $\mathrm{g}$ is a complete elipse. On the other hand, lowercase $c$ and $e$ are more similar in the type font used for the paper condition, in which the curls in slightly at the top (as though it were starting a feature for the letter e), than in that used in the CRT condition, in which there is no inward curl at the top of the $c$ and the curve at the bottom of the $c$ extends somewhat higher than does the one at the bottom of the e.

Finding an influence of type font on the pattern of proofreading errors in the CRT and paper conditions does not in any way diminish the fact that the proofreading error patterns in both conditions are consistent with the hierarchical feature test and the asymmetrical decision rule. In other words, despite the facts that the text was presented under vastly different perceptual conditions, that the task demands of the two procedures differed to some extent (e.g., there was some time pressure placed on the subjects in the CRT condition but not on those in the paper condition), and that type font varied, the same information processing rules were implicated in the two situations. Differences in type font also proved to be of minor importance in the earlier study by Healy (1981). In Experiment 3 of that study, type font was varied and this variation was not confounded with a difference in perceptual condition: Subjects proofread photocopies of typewritten passages that were printed with an Elite 12 print wheel (as in the present study) or with a Prestige Elite 12 print wheel. The most notable difference between these two type fonts is that the c curls in slightly at the top in the Elite 12 font but there is no inward curl of the $c$ and the gap in the $c$ is somewhat larger than the gap in the $e$ in the Prestige Elite font. Analogous patterns of proofreading errors were found for the two print wheels in that study.

False alarms. The false-alarm rate (responses to correctly spelled words) was not high; 126 false-alarm errors were made in total, which averages 2.6 per passage. The false-alarm rate was higher in the CRT condition (91 false-alarm errors) than in the paper condition 
(35). The difference between the two conditions is presumably due to the presence in the CRT condition of some late responses that were made during the presentation of the subsequent five-word segment and thus scored as false-alarm errors. The existence of late response errors is supported by finding a larger number of false-alarm errors (27) among the 2 subjects in the CRT condition who experienced a display duration of 2,500 msec instead of 3,000 msec, which is the reason why the duration was increased for the 10 remaining subjects.

Response latencies. The mean latencies for correct responses showed a pattern consistent with that found for proofreading errors, although the pattern was less regular. The general agreement in results concerning proofreading errors and latencies suggests that speed/ accuracy tradeoffs are not likely to be playing a major role, if any, in this task. The irregularity in the response times may be due to the fact that a given latency is undoubtedly affected by the location within a fiveword segment of the misspelled word, but this factor was not controlled in the present experiment. For this reason, the latencies will not be considered here in detail.

\section{EXPERIMENT 2}

The similarity in the patterns of proofreading errors for the two perceptual conditions of Experiment 1 points to the generality of the information processing strategies used by proofreaders. Proofreaders employ the asymmetrical decision rule even in the CRT condition, in which the rule cannot easily be explained in terms of subjects' expectations concerning missing segments resulting from a poor photocopy process. The question remains, however, whether subjects' strategies can be modified at all by perceptual conditions. Will the asymmetrical decision rule be employed even when subjects are led to expect additional line segments rather than missing ones?

In order to investigate the persistence of the proofreading strategies, four different perceptual conditions were compared, each of which made use of ditto copies (purple lettering on a white background): (1) the "highquality" condition, which involved relatively clear-cut copies with printing that was nonetheless somewhat less distinct than that resulting from the photocopy process used in Experiment 1, (2) the "hyphen" condition, which was the same as the high-quality condition except for the addition of extraneous hyphens superimposed on some of the characters, (3) the "slash" condition, which was the same as the hyphen condition except that slashes replaced the hyphens, and (4) the "low-quality" condition, which employed ditto copies with printing that was quite blurred and with extraneous random noise that was naturally generated by the ditto process.

The hyphen and slash conditions resembled the process limitation condition used by Garner and Haun (1978) except that our conditions involved only letter- feature additions, whereas their condition involved both additions and deletions of letter features. Although none of the extraneous noise characters added in our conditions (hyphens or slashes) were superimposed on any letters of misspelled words, the presence of these additional line segments might lead subjects to tolerate misspellings involving letter substitutions that add letter features (e.g., when $\mathrm{c}$ is replaced by e). Perhaps subjects would tolerate any additional letter feature whenever extraneous line segments are superimposed on the text. If so, then subjects should give up the asymmetrical decision rule in both the hyphen and slash conditions for all three letter pairs (c/e, c/g, and c/o). Alternatively, subjects may tolerate additional letter features only when the features resemble the extraneous noise characters. In that case, subjects should retain the asymmetrical decision rule in the slash condition, since the slash does not resemble any of the relevant letter features, but they should give up the asymmetrical rule in the hyphen condition and tolerate additional horizontal features that resemble the hyphen (as when e replaces $\mathrm{c}$ or $\mathrm{G}$ replaces $\mathrm{C}$ but not when o replaces $\mathrm{c}$ or $\mathrm{O}$ replaces $\mathrm{C}$ ).

Extraneous noise characters also occurred in the lowquality condition, but these characters differed from those in the hyphen and slash conditions in two respects: They were not homogeneous in size and orientation, and they were not placed systematically onto the words of the text. As in the hyphen and slash conditions, the low-quality condition differed from the highquality condition only in the addition, not in the deletion, of line segments. If subjects tolerate added letter features whenever the features can be mistaken for extraneous noise, then subjects in the low-quality condition should give up the asymmetrical decision rule in favor of a strategy that tolerates both added and deleted letter features of any orientation, since the letter features may not be distinguishable from the random noise created by the copying procedure.

\section{Method}

Subjects. Forty-eight undergraduate men and women from the University of Colorado participated for introductory psychology course credit.

Materials and apparatus. The prose passage used in Experiment 1 was also employed here in its two versions, lowercase and uppercase. Different forms of the typecase versions were created for the four perceptual conditions. The forms for the highquality condition were equivalent to those for the paper condition of Experiment 1 except that the text was typed in paragraph format rather than in five-word columns, and ditto copies rather than photocopies were employed.

The forms for the hyphen condition were the same as those for the high-quality condition except that hyphens were superimposed on selected letters. Placement of the hyphens was determined in the following manner: The prose passage, excluding the first word, was divided into groups of three consecutive words. A hyphen (approximately midletter in position) was superimposed on one letter in each group of three words pseudorandomly, with the only restriction being that it could not appear on any letters of a misspelled word. Thus, the misspelled words in the hyphen condition were identical in legibility to those in the high-quality condition. The forms for the slash condition were the same as those for the hyphen condition 
except that slashes replaced the hyphens. The ditto copies were made by inserting the typewritten originals from the Diablo 1620 printer into a copier/transparency maker, Standard Model 8700 , to obtain a master copy for use with the ditto machine. The forms for the low-quality condition were created in the same manner as those for the high-quality condition except for an adjustment on the pressure dial of the copier/transparency maker that increased the ink flow and allowed for the creation of ditto copies with random speckles and an increased broadening of the print (without any increase in letter-part deletions). Note that the misspelled words in the low-quality condition were not as legible as those in the high-quality, hyphen, or slash conditions, since they were not protected from the extraneous noise as they were protected from the hyphens and slashes in the other conditions.

Each typecase version of the passage was two pages long and was typed with single spacing, with an extra blank line separating paragraphs. The typing was accomplished using the same apparatus as in Experiment 1. Ditto copies, white background with purple letters, were made for distribution to the subjects.

Subjects made their responses in all conditions by writing with a pen or pencil.

Design. The same four-way mixed design was employed as in Experiment 1 except that there were four levels of the betweensubjects factor (high quality, hyphen, slash, low quality), instead of two. Twelve different subjects were tested in each condition. The presentation order of the typecase versions was counterbalanced within each condition. The assignment of subjects to conditions and presentation orders was determined on the basis of time of arrival for testing according to a fixed rotation.

Procedure. Subjects were tested in small groups ranging from one to six individuals. Ditto, typewritten instructions of high quality were distributed to the subjects. Subjects in all conditions were told to read each passage at their normal reading speed and to circle any spelling errors that they encountered. Subjects were also told not to retrace their steps if they thought they had missed a spelling error and not to refer to the first passage when proofreading the second. Subjects in the hyphen condition were informed that hyphens were superimposed on some of the letters but that the hyphens were "noise" characters to be ignored and their presence did not constitute a misspelling. Subjects in the slash condition received similar instructions concerning the slashes. The instructions in the low-quality condition were the same as those in the high-quality condition; subjects were not forewarned of the poor quality of the printing. As soon as the subjects in a group began proofreading, the experimenter commenced timing them with a stopwatch. After proofreading both passages, subjects raised their hands, and the experimenter recorded the time for each individual from the watch.

\section{Results and Discussion}

Misses. The number of misspellings not circled for each type of misspelling in each passage typecase was computed for each subject. The scores from both presentation orders were combined, and the mean percentages of proofreading errors were computed as a function of condition, passage typecase, and substitution type. These values are displayed in Table 4 . The standard errors of the values, as determined by analyses of variance, are $3.2 \%$ for the high-quality condition, $3.4 \%$ for the hyphen condition, $3.2 \%$ for the slash condition, and $3.6 \%$ for the low-quality condition.

A multifactorial analysis of variance, analogous to that used in Experiment 1, was conducted. The significant results of the analysis are summarized in Table 5 .

The overall pattern of results favored the hierarchical feature test and the asymmetrical decision rule in this experiment as in the previous studies, but there were also some dramatic effects of perceptual condition in this study. Furthermore, this experiment for the first time yielded significantly more proofreading errors on the lowercase version $(45.7 \%)$ than on the uppercase version (34.9\%), which may have resulted simply because the poor quality of the print brought out the advantage in terms of discriminability of larger characters.

Support for the hierarchical feature test was provided by the significant interaction of passage typecase and letter pair. Both letter pairs $\mathrm{c} / \mathrm{e}$ and $\mathrm{c} / \mathrm{o}$ showed more proofreading errors in lowercase (c/e: $56.4 \%$; c/o: $57.1 \%$ ) than in uppercase (C/E: $27.2 \%$; C/O: 33.1\%), whereas the pair $\mathrm{c} / \mathrm{g}$ showed more proofreading errors in uppercase $(44.3 \%)$ than in lowercase $(23.5 \%)$. The greater error rate for $\mathrm{c} / \mathrm{o}$ in the lowercase version may be due to the fact that the gap in the letter $c$ was somewhat smaller in the lowercase type than in the uppercase type, so that the letters $c$ and o may be somewhat harder to discriminate in lowercase. Although the c/o pair showed a greater disadvantage for the lowercase version than had been found in previous experiments, the trend is clearly consistent with the observation from earlier studies that subjects made more proofreading errors when letter envelope was maintained than when it was changed.

The asymmetrical decision rule was supported by the significant overall difference in proofreading error rates

Table 4

Percentages of Proofreading Errors in Experiment 2 as a Function of Perceptual Condition, Passage Type Case, and Substitution Type

\begin{tabular}{|c|c|c|c|c|c|c|c|}
\hline \multirow[b]{2}{*}{ Perceptual Conditior } & & \multicolumn{6}{|c|}{ Substitution Type } \\
\hline & & $\mathrm{c}$ to $\mathrm{e}$ & e to $c$ & $c$ to g & $\mathrm{g}$ to $\mathrm{c}$ & $\mathrm{c}$ to $\mathrm{o}$ & o to $\mathrm{c}$ \\
\hline High Quality & $\begin{array}{l}\text { Lowercase } \\
\text { Uppercase }\end{array}$ & $\begin{array}{l}31.1 \\
17.8\end{array}$ & $\begin{array}{l}49.4 \\
21.7\end{array}$ & $\begin{array}{l}13.9 \\
28.9\end{array}$ & $\begin{array}{l}18.9 \\
47.2\end{array}$ & $\begin{array}{l}23.3 \\
13.9\end{array}$ & $\begin{array}{l}63.9 \\
31.7\end{array}$ \\
\hline Hyphen & $\begin{array}{l}\text { Lowercase } \\
\text { Uppercase }\end{array}$ & $\begin{array}{l}46.1 \\
17.8\end{array}$ & $\begin{array}{l}52.2 \\
22.2\end{array}$ & $\begin{array}{l}13.3 \\
40.6\end{array}$ & $\begin{array}{l}25.0 \\
43.3\end{array}$ & $\begin{array}{l}30.0 \\
21.7\end{array}$ & $\begin{array}{l}63.9 \\
50.0\end{array}$ \\
\hline Slash & $\begin{array}{l}\text { Lowercase } \\
\text { Uppercase }\end{array}$ & $\begin{array}{l}38.9 \\
22.8\end{array}$ & $\begin{array}{l}62.8 \\
25.0\end{array}$ & $\begin{array}{l}18.3 \\
38.9\end{array}$ & $\begin{array}{l}24.4 \\
47.2\end{array}$ & $\begin{array}{l}35.6 \\
21.1\end{array}$ & $\begin{array}{l}67.2 \\
33.9\end{array}$ \\
\hline Low Quality & $\begin{array}{l}\text { Lowercase } \\
\text { Uppercase }\end{array}$ & $\begin{array}{l}91.1 \\
53.9\end{array}$ & $\begin{array}{l}79.4 \\
36.7\end{array}$ & $\begin{array}{l}29.4 \\
50.0\end{array}$ & $\begin{array}{l}45.0 \\
58.3\end{array}$ & $\begin{array}{l}79.4 \\
52.2\end{array}$ & $\begin{array}{l}93.3 \\
40.6 \\
\end{array}$ \\
\hline
\end{tabular}


Table 5

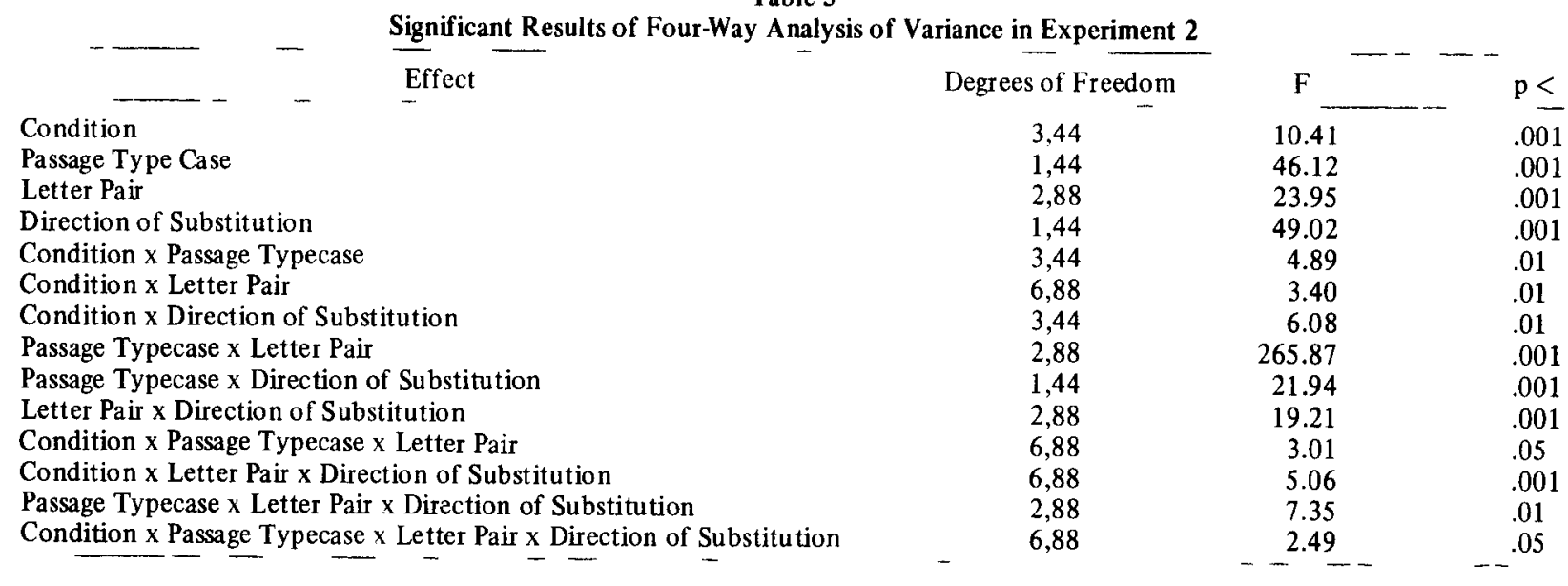

between feature deletions (46.0\%) and feature additions $(34.6 \%)$ and by the fact that the difference between deletions and additions was greatest when letter envelope was maintained (for $\mathrm{c} / \mathrm{e}, \mathrm{C} / \mathrm{G}, \mathrm{c} / \mathrm{o}$, and $\mathrm{C} / \mathrm{O}$ ), as reflected in the significant three-way interaction of passage typecase, letter pair, and direction of substitution. However, the asymmetrical decision rule was not consistently applied in all conditions.

The factor of perceptual condition yielded a significant main effect as well as a number of significant interactions, including the four-way interaction of condition, passage typecase, letter pair, and direction of substitution. Almost twice as many proofreading errors were made in the low-quality condition $(59.1 \%)$ as in the other conditions (high quality: $30.1 \%$; hyphen: $35.5 \%$; slash: $36.3 \%$, which is reasonable given the differences in legibility. Separate analyses of variance were conducted on the data from each condition in order to evaluate the different patterns of results. As expected, the highquality and slash conditions yielded results consistent with those from earlier experiments and with the use of the hierarchical feature test and asymmetrical decision rule. The hierarchical feature test was supported by significant interactions of passage typecase and letter pair [high quality: $F(2,22)=51.38, p<.001$; slash: $F(2,22)=97.04, p<.001]$. As with the overall data, subjects made more proofreading errors on $\mathrm{c} / \mathrm{e}$ in lowercase than in uppercase; subjects also made more proofreading errors on $\mathrm{c} / \mathrm{o}$ in lowercase than in uppercase; but subjects made more proofreading errors on $\mathrm{c} / \mathrm{g}$ in uppercase than in lowercase. The asymmetrical decision rule was supported by the greater proofreading error percentages with deleted features than with added features, as reflected in the significant main effect of direction of substitution [high quality: $\mathrm{F}(1,11)=22.09$, $p<.001$; slash: $F(1,11)=17.16, p<.01]$, and by the significant three-way interaction of passage typecase, letter pair, and direction of substitution [high quality: $F(2,22)=8.72, p<.01 ;$ slash: $F(2,22)=4.14, p<.05]$.

A new pattern of results was obtained in the hyphen condition, which suggested that the subjects employed the hierarchical feature test coupled with a decision rule that tolerated missing features of any orientation and tolerated added features with a horizontal orientation only. The hierarchical feature test was supported by the significant interaction of passage typecase and letter pair $[F(2,22)=42.68, p<.001]$. Once again, subjects made more proofreading errors on c/e and c/o substitutions in lowercase than in uppercase, but they made more proofreading errors on $\mathrm{c} / \mathrm{g}$ in uppercase than in lowercase. The new decision rule was supported by a significant overall difference between deleted features and added features $[F(1,11)=16.29, p<.01]$, which was qualified by a significant interaction of letter pair and direction of substitution $[\mathrm{F}(2,22)=11.68, \mathrm{p}<.001]$. The difference between deleted and added features was very large for $\mathrm{c} / \mathrm{o}$ but was negligible for $\mathrm{c} / \mathrm{e}$ and for $\mathrm{c} / \mathrm{g}$. The three-way interaction of passage typecase, letter pair, and direction of substitution was not significant in the hyphen condition $[F(2,22)<1]$.

A different pattern of results was obtained with the low-quality condition, and this pattern suggested that in this case subjects continued to employ the hierarchical feature test but combined this test with a decision rule that tolerated both added and deleted features of all orientations. The hierarchical feature test was supported by the strong interaction of passage typecase and letter pair $[F(2,22)=110.72, p<.001]$. Subjects continued to make more proofreading errors on $c / e$ and $\mathrm{c} / \mathrm{o}$ in lowercase than in uppercase, and they made more proofreading errors on $\mathrm{c} / \mathrm{g}$ in uppercase than in lowercase. The new decision rule was implicated by finding proofreading errors on added features to be just as frequent as those on deleted features $[F(1,11)<1]$. Furthermore, the three-way interaction of passage typecase, letter pair, and direction of substitution was not significant $[F(2,22)=2.38, p>.10]$, although there was a significant two-way interaction of passage typecase and direction of substitution $[F(1,11)=8.79, p<.05]$ and a significant two-way interaction of letter pair and direction of substitution $[F(2,22)=15.84, p<.001]$, which were not predicted and have yet to be explained. 
Proofreading times. Despite the overall difference in proofreading errors among the perceptual conditions, there was not a difference in reading times $[F(3,44)=$ $1.41, \mathrm{p}>.10 \mathrm{l}$. The mean proofreading times were $15.3,16.3,17.8$, and $16.7 \mathrm{~min}$ for the high-quality, hyphen, slash, and low-quality conditions, respectively. Although the proofreading error rate was high in the low-quality condition, the reading time was not significantly longer in that condition than in the other conditions.

False alarms. The false-alarm rate was somewhat higher in this experiment than in the previous one. The 48 subjects made 306 false alarms in total, an average of 3.2 per passage. A two-way mixed-design analysis of variance was performed for these data including the between-subjects factor of perceptual condition and the within-subjects factor of passage typecase.

As found for misses, the lowercase version yielded significantly more false-alarm errors (mean $=3.7$ ) than the uppercase $(2.6)[F(1,44)=4.25, \mathrm{p}<.05]$. A significant interaction of perceptual condition and passage typecase also resulted. The greatest number of false alarms occurred in the lowercase version of the hyphen condition, presumably because subjects were likely to mistake the hyphen for a letter feature in that condition. In the other conditions and passage versions, the mean number of false alarms did not differ markedly (high quality-lowercase: 2.2 , uppercase: 1.7 ; hyphenlowercase: 6.5 , uppercase: 2.6 ; slash-lowercase: 1.9 , uppercase: 3.0 ; low quality-lowercase: 4.3 , uppercase: 3.3). The main effect of perceptual condition was not significant $[F(3,44)=1.57, p>.10]$. In particular, it is important to note that although subjects made more misses in the low-quality condition, they did not make more false alarms in that condition (mean $=3.8$ ) relative to the other conditions (high quality: 1.9; hyphen: 4.5 ; slash: 2.5 ).

\section{GENERAL DISCUSSION}

Different perceptual conditions were compared in this study in order to assess the generality and persistence of the information processing strategies used by proofreaders when searching for misspelled words. Earlier experiments by Healy (1981) had suggested that proofreaders employ a hierarchical feature test that gives first priority to resolving letter envelope and second priority to resolving other letter features. Furthermore, for those misspellings that do not alter envelope, a sophisticated-guessing decision rule was implicated by the finding that subjects tolerated misspellings that resulted in missing letter features but not those that added features. One possible rationale for the use of the sophisticated-guessing rule is that, in reading copies of typewritten text, subjects may expect to find missing line segments resulting from a poor photocopying process. According to this line of reasoning, proofreaders may give up the sophisticated-guessing rule under per- ceptual conditions in which they do not expect to find missing segments or they are led to expect added line segments as well as deleted ones. In order to test this hypothesis in Experiment 1, we compared proofreading typewritten text with proofreading text presented on a CRT screen, since subjects should have no reason to expect missing segments in the screen condition. The generality of the sophisticated-guessing strategy was demonstrated by its occurrence in both perceptual conditions, although there were some differences in the detailed pattern of proofreading errors under the two conditions that seemed to result from differences in type font.

The persistence of the sophisticated-guessing strategy was demonstrated in the slash condition of Experiment 2 by the finding that subjects used it even when they were led to expect added extraneous line segments that were similar to but not the same as the critical letter features. On the other hand, in the hyphen and low-quality conditions of Experiment 2, the added noise characters could not be distinguished from the critical letter features, and in those conditions subjects were found to modify their decision rule so that they tolerated all missing letter features and any added letter features that resembled the noise. The finding of a switch in strategy in the low-quality condition of Experiment 2 is particularly important because the superimposed noise in that condition was not artificial but, rather, was the natural product of the ditto process, and the copies proofread by subjects resembled those often found in the classroom. The results of this condition make it clear that adequate printing quality, not low quality, is responsible for the asymmetrical decision rule.

Although the results of the low-quality condition may be the most useful from a practical standpoint, they are subject to an interpretive problem that is not applicable to the other conditions. The noise characters present in the low-quality condition were not excluded from the misspelled words. Therefore, we cannot be certain that the subjects tolerated added features in that condition because of the added noise in the surrounding text or simply because the misspelled words themselves were not entirely legible. However, in the hyphen and slash conditions, the misspelled words were specifically protected from the noise segments (see the Method section of Experiment 2), so that the misspelled words in the hyphen condition were just as crisp in quality as those in the slash and high-quality conditions. Finding a tolerance of additional horizontal line segments in the hyphen condition of Experiment 2 but not in the slash or high-quality conditions of the same experiment therefore provides strong support for the hypothesis that subjects used a different information processing rule in the hyphen condition.

The results of the hyphen condition are particularly instructive for three other reasons as well: First, the specificity of the information processing rule is demonstrated by the fact that subjects made almost as many 
proofreading errors for added features as for deleted features with $\mathrm{c} / \mathrm{e}$ and $\mathrm{C} / \mathrm{G}$ but made many fewer proofreading errors for added features than for deleted features with $\mathrm{c} / \mathrm{o}$ and $\mathrm{C} / \mathrm{O}$. It seems that only when the added letter features resembled the noise characters, which were horizontal lines in the hyphen condition, did the subjects tolerate them. Further support for specificity comes from the slash condition. Although noise characters were added in that condition in the same manner as in the hyphen condition, subjects showed no tolerance of added letter features there, presumably because the added slashes did not resemble any of the critical features.

Second, the pattern of proofreading errors in the hyphen condition of Experiment 2 was different from that in the same condition of an unpublished preliminary experiment, which differed only in the quality of the printing; in the preliminary experiment, clear-cut offset press copies were used in which the hyphen was similar to, but clearly distinguishable from, the horizontal letter features, whereas in Experiment 2 ditto copies were used in which the characters were less clearcut so that it was difficult to distinguish the hyphens from the horizontal letter features. Although the two hyphen conditions yielded similar results for the substitutions involving $C$ and $G$, the subjects showed the usual strong asymmetry for substitutions involving $c$ and $e$ in the hyphen condition of the preliminary experiment but not in the corresponding condition of Experiment 2. It thus seems that subjects will tolerate added letter features only when they cannot distinguish those features from extraneous noise. This finding demonstrates the importance of the crispness of the printing quality in determining the subjects' information processing rules.

Third, since accuracy was quite low in the lowquality condition, one could argue that any asymmetry in the pattern of proofreading errors may have been masked to a certain extent by the presence of a ceiling on proofreading errors, especially in the same-envelope cases. However, such a ceiling effect cannot be used to explain the lack of asymmetry in the hyphen condition because the proofreading error rate averaged below $50 \%$ in that condition even in the same-envelope cases, and the overall proofreading error rate for the hyphen condition was no greater than that in the slash condition, in which the usual asymmetries were evident.

It could be argued that some of the proofreading errors we found may be attributed to the subjects' lack of knowledge concerning the correct spelling of the words, rather than to the information processing rules they used. However, in the unpublished preliminary experiment, we administered both our proofreading task and a spelling test to 72 subjects. The spelling test, which was conducted after the subjects had completed proofreading both typecase versions of our passage, included the 90 misspelled words along with their correct spellings. The 90 pairs of words were listed in their order of appearance in the prose passage, and the correctly spelled word preceded the misspelling in half of the pairs. Subjects were told to circle the misspelled word in each pair. We found the typical pattern of proofreading errors in this experiment, but only 11 (of 6,480 possible) mistakes on the spelling test. It therefore seems unlikely that spelling knowledge can account for the pattern of proofreading errors we found.

Although we found that subjects did not use the same decision rule in all perceptual conditions of our experiments, the hierarchical feature test was employed in each case, as witnessed by the fact that subjects made more proofreading errors on substitutions in which letter envelope was maintained than on those in which envelope was changed. Furthermore, subjects never gave up their tolerance of missing features. Rather, when changing their decision rule, as they did in the hyphen and low-quality conditions of Experiment 2, they merely added a tolerance of extra features. Therefore, sophisticated guessing can still be used to describe their decision rule, despite the lack of asymmetry, as long as it is understood that only those letter features not attributable to noise serve as constraints on the letter recognition responses.

What are the theoretical implications of the information processing rules supported by these experiments? These rules allow the proofreader to identify words in a more economical and efficient fashion than would be possible if each letter feature were given equal weight and its presence had to be separately verified. Peripheral vision may suffice for resolving those features defining letter envelope, but foveal vision may be necessary for resolving additional letter features. Hence, the hierarchical feature test may permit the proofreader to make greater use of peripheral visual information than would a rule giving equal weight to all letter features. Furthermore, the sophisticated-guessing rule may permit the proofreader to identify a given letter without finishing the verification process; the proofreader need not wait until the presence of each constituent letter feature has been confirmed. If the proofreader identifies a letter before checking for the presence of all of its features, then a missing feature (like the horizontal segment in e when it is replaced by c) can be ignored because it can be interpreted as a feature that is present in the identified letter (e) but not yet confirmed, whereas an additional feature (like a horizontal segment added to $c$ when it is replaced by e) cannot be ignored (unless it is attributed to noise) because it is inconsistent with the identified letter (c). These information processing rules, taken together, are also compatible with a unitization process (see, e.g., Healy \& Drewnowski, 1983), in which common words may be identified without the complete identification of each of their component letters. The features defining letter envelope are most likely to aid in the identification of supraletter units such as frequently occurring syllables or words.

Why did we find evidence for sophisticated guessing in 
these experiments, whereas such evidence was lacking from earlier studies involving tachistoscopic letter report (see Massaro \& Schmuller, 1975)? Perhaps readers are able to adopt such a strategy only when they can use top-down contextual information as well as bottomup featural information in making their identifications. Such contextual information was clearly available in the prose passage used in the present study but was absent from, or minimal in, previous studies of alphabetic confusions, which involved the presentation of single letters (e.g., Townsend, 1971), letter pairs (e.g., Podgorny \& Gamer, 1979), or short words (e.g., Bouwhuis \& Bouma, 1979). Furthermore, such a strategy may be evident only when subjects view more than one letter at a time, just as it has recently been found (see Healy, Oliver, \& McNamara, Note 1) that letterdetection errors occur predominantly in common words only when more than one word can be seen at once. Perhaps subjects are driven to process a subsequent letter when one is available once they have been able to identify the current letter, even if the presence of all of the constituent letter features in the current letter have not been verified. In contrast, when only one letter is in view at a time, subjects may continue processing that letter even after it has been identified, since no other letters are available for processing.

These experiments, which demonstrate the reliance of processing strategy on perceptual conditions, underline the fact that in the laboratory we should attempt to replicate those conditions that are most common in everyday life, so that the information processing strategies we identify are most likely to be those actually employed outside the experimental setting.

\section{REFERENCE NOTE}

1. Healy, A. F., Oliver, W. L., \& McNamara, T. P. Detecting letters in continuous text: Effects of display size. Paper presented at the 23rd annual meeting of the Psychonomic Society, Minneapolis, Minnesota, November 1982.

\section{REFERENCES}

Aronson, E. The social animal. San Francisco: Freeman, 1972.

Brondbent, D. E. Word-frequency effect and response bias. Psychological Review, 1967, 74, 1-15.

Bouma, H. Visual recognition of isolated lower-case letters. Vision Research, 1971, 11, 459-474.

Bouwhuis, D., \& Bouma, H. Visual word recognition of threeletter words as derived from the recognition of the constituent letters. Perception \& Psychophysics, 1979, 25, 12-22.

GARNER, W. R. The role of configuration in the identification of visually degraded words. Memory \& Cognition, 1981, 9, 445-452.

Garner, W. R., \& HaUn, F. Letter identification as a function of type of perceptual limitation and type of attribute. Journal of Experimental Psychology: Human Perception and Performance, $1978,4,199-209$.

Healy, A. F. Proofreading errors on the word the: New evidence on reading units. Journal of Experimental Psychology: Human Perception and Performance, 1980, 6, 45-57.

Healy, A. F. The effects of visual similarity on proofreading for misspeilings. Memory \& Cognition, 1981, 9, 453-460.

Healy, A. F., \& Drewnowski, A. Investigating the boundaries of reading units: Letter detection in misspelled words. Journal of Experimental Psychology: Human Perception and Performance, 1983, 9, 413-426.

Ноцвоок, M. B. Effect of subjective interletter similarity, perceived word similarity, and contextual variables on the recognition of letter substitutions in a proofreading task. Perceptual and Motor Skills, 1978, 47, 251-258.

Koffka, K. Principles of gestalt psychology. New York: Harcourt, Brace, 1935.

Kučera, H., \& Francis, W. N. Computational analysis of presentday American English. Providence, R.I: Brown University Press, 1967

Lindsay, P. H, \& Norman, D. A. Human information processing: An introduction to psychology (2nd ed.). New York: Academic Press, 1977.

LUPKER, S. J. On the nature of perceptual information during letter perception. Perception \& Psychophysics, 1979, 25, 303-312.

Massaro, D. W, \& Schmuller, J. Visual features, preperceptual storage, and processing time in reading. In D. W. Massaro (Ed.), Understanding language: An information processing analysis of speech perception, reading, and psycholinguistics. New York: Academic Press, 1975.

Podgonny, P., \& Garner, W, R. Reaction time as a measure of inter- and intraobject visual similarity: Letters of the alphabet. Perception \& Psychophysics, 1979, 26, 37-52.

Rumelhart, D. E., \& Siple, P. Process of recognizing tachistoscopically presented words. Psychological Review, 1974, 81, 99-113.

Townsend, J. T. Theoretical analysis of an alphabetic confusion matrix. Perception \& Psychophysics, 1971, 9, 40-50.

\section{NOTES}

1. For simplicity and ease of analysis, we define a letter feature as a short line segment (see Rumelhart \& Siple, 1974), although we acknowledge that alternative formulations (such as those allowing gaps as features) are reasonable and should be addressed in future studies.

2. An analysis of variance conducted with only the data from the 10 subjects in the CRT condition with the $3,000-\mathrm{msec}$ display duration yielded results similar to those from the analysis with the data from all 12 subjects, except that in the reduced analysis the expected three-way interaction of direction of substitution, passage typecase, and letter pair did reach statistical significance $[F(2,18)=6.95, \mathrm{p}<.01]$.

(Manuscript received July 1,1982; revision accepted for publication July 11,1983 .) 